Scatter in an uncollimated $x$-ray CT machine based on a Geant4 Monte Carlo simulation

Wadeson, Nicola and Morton, Edward and Lionheart, William

2010

MIMS EPrint: 2010.66

Manchester Institute for Mathematical Sciences

School of Mathematics

The University of Manchester

\footnotetext{
Reports available from: http://eprints.maths.manchester.ac.uk/

And by contacting: The MIMS Secretary

School of Mathematics

The University of Manchester

Manchester, M13 9PL, UK
} 


\title{
Scatter in an uncollimated x-ray CT machine based on a Geant4 Monte Carlo simulation.
}

\author{
Nicola Wadeson ${ }^{a}$, Edward Morton ${ }^{b}$ and William Lionheart ${ }^{a}$ \\ ${ }^{a}$ School of Mathematics, University of Manchester, Manchester, UK; \\ ${ }^{b}$ Rapiscan Systems Ltd
}

\begin{abstract}
A high-speed motionless-gantry x-ray CT machine has been designed to allow for 3D images to be collected in real time. By using multiple, switched x-ray sources and fixed detector rings, the time consuming mechanical rotation of conventional CT machines can be removed. However, the nature of this design limits the possibility of detector collimation since each detector must now be able to record the energy of x-ray beams from a number of different directions. The lack of collimation has implications in the reconstructed image due to an increase in the number of scattered photons recorded. A Monte Carlo computer simulation of the x-ray machine has been developed, using the Geant4 software toolkit, to analyse the behaviour of both Rayleigh and Compton scattered photons when considering airport baggage and medical applications. Four different scattering objects were analysed based on $50 \mathrm{kVp}, 100 \mathrm{kVp}$ and $150 \mathrm{kVp}$ spectra for a tungsten target. Two suitcase objects, a body and a brain phantom were chosen as objects typical of airport baggage and medical CT. The results indicate that the level of scatter is negligible for a typical airport baggage application, since the majority of space in a suitcase consists of clothing, which has a low density. Scatter contributes to less than $1 \%$ of the image in the $100 \mathrm{kVp}$ and $150 \mathrm{kVp}$ instances. However, due to the large amounts of water found in the human body, the level of scatter in the medical instances are significantly higher, reaching $37 \%$ when the body phantom is analysed at $50 \mathrm{kVp}$.
\end{abstract}

Keywords: Compton scatter, Rayleigh scatter, Geant4, Monte-Carlo simulation, x-ray CT, RTT.

\section{INTRODUCTION}

A motionless gantry real-time tomography (RTT) machine is being developed ${ }^{1}$ to examine the 3D images of airport baggage in real time. The RTT system (Fig. 1) uses multiple, switched x-ray sources and fixed detector rings in order to eliminate the time consuming mechanical rotation of conventional CT machines.

In a vacuum an x-ray beam travels in a straight line. However, when an x-ray beam is projected in a straight line through an object some of the photons in the beam are lost. At the energies used in this application $(30-160 \mathrm{keV})$, this is due to either photoabsorption or x-ray scatter. The scattered photons can be sent in any direction, according to some probability, and are unlikely to follow the same line as the original beam. When the energy of the beam is recorded in a detector after exiting the object, the resulting energy will be lower due to this attenuation.

Most of the scattered photons will be scattered out of plane of the detectors. However, some scattered photons may deviate from their original beam path and be recorded in another detector, falsely increasing the energy of a different x-ray beam. This leads to degradation of the output image. It has been shown that scatter leads to streak artifacts in the image and adds to the cupping artifacts which are also attributed to beam hardening. ${ }^{3-5,9}$ The total amount of scatter contributing to the image is dependent upon the object material properties (atomic and electron densities and atomic number), object size and distance to detectors, as well as incident photon energy. If the ratio of the scattered photons to the incident photons is high, this could seriously degrade the image.

Further author information:

E-mail: nicola.wadeson@maths.manchester.ac.uk 


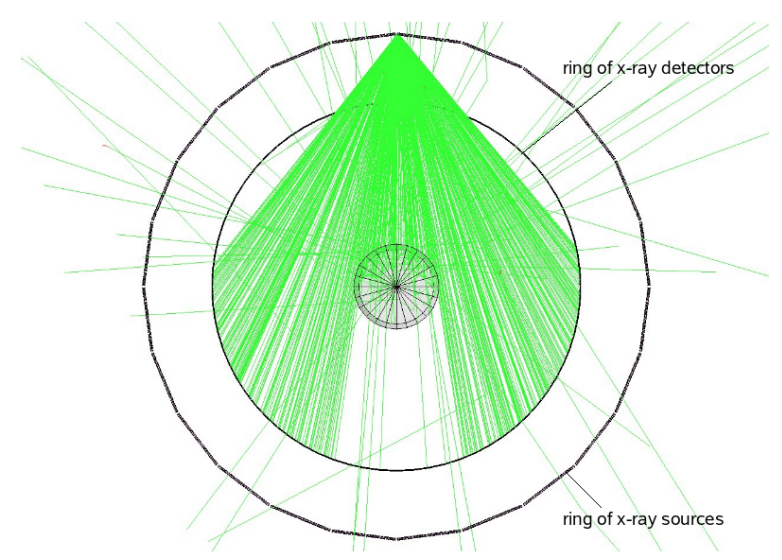

Figure 1: The RTT x-ray machine geometry setup with fixed source and detector rings, which are offset in the z-direction, showing one source projection of x-ray beams. Since the detector ring is fixed, each detector must be able to record the energy of x-ray beams from a number of different directions so there is no in plane detector collimation.

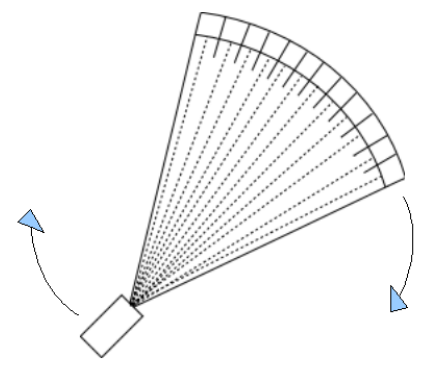

Figure 2: A rotating source and collimated detector segment, typical of conventional x-ray CT machines.

In conventional x-ray CT machines, with a rotating source and detector gantry (Fig. 2), each detector is collimated to limit the angle of scattered photons that can reach a detector. However, in the RTT system, since the detector ring is fixed, the same detector must be able to collect photons from a number of different source projections travelling from a number of different angles, so this type of collimation is not possible.

\section{X-RAY SCATTER}

In $\mathrm{x}$-ray $\mathrm{CT}$ it is desired to reconstruct the attenuation coefficient $f(\mathbf{x})$ of a material by considering line integrals through the object, where $f(\mathbf{x})$ is a measure of how much energy the $\mathrm{x}$-ray beam has lost at a point $\mathbf{x}$ and is proportional to the density of the object. The three attenuating processes are photoabsorption and the two scattering processes, Compton scatter and Rayleigh scatter. In practice, however, what is actually reconstructed is $f(\mathbf{x})+S$, where $S$ is attributed to detected scatter and other noise such as statistical variations in the data. A photon can be scattered multiple times making it difficult to determine where the scattering event occured, even if the angle of incidence on the detector is known.

Compton scattering is a incoherent scattering process that occurs when a photon collides with an atomic electron and imparts some of its energy to the electron. The resulting lower energy photon continues in a different direction. Since the number of Compton scattering events is dependent upon the number of electrons present in a material, the Compton scattered photons provide information about the electron density of the scattering material. 
Rayleigh scattering is an coherent scattering process that occurs when a photon collides with an atom, causing the photon to deviate from its original path without any loss of energy. The Rayleigh scattered photons provide information about the atomic structure of the scattering material and are utilised in diffraction techniques in order to identify specific materials, particularly those of a crystalline nature. ${ }^{2,6}$

The probability of photoabsortion decreases with increasing incident photon energy, causing Compton scatter to become the dominant process at higher energies. Rayleigh scatter decreases with increasing energy, but is expected to dominate over Compton scatter at very low angles of less than 10 degrees. Scattering can occur in both the forward and backward directions and provides a smooth contribution to the image.

\section{GEANT4 SIMULATION}

Geant4 is a software toolkit, ${ }^{7}$ based on Monte-Carlo methods, that simulates the passage of particles through matter. The RTT geometry was incorporated into the existing Geant4 framework along with the required physics processes - photoelectric effect, Compton scatter and Rayleigh scatter - to create the RTTModel. The RTTModel allows the energy recorded in each detector to be separated into energy contributions from primary particles, Rayleigh and Compton scattered particles and particles that have been scattered multiple times.

The RTTModel uses the Geant4 low energy Compton Scattering package (G4LECS) to model the Compton and Rayleigh scattering processes. ${ }^{8}$ This extension package is more accurate than the standard G4 processes as it takes atomic binding into account, which leads to Doppler broadening effects. These effects are important at low energies such as those used in this application. A slight variation on the standard Compton formula is used to introduce the Doppler broadening effects. ${ }^{10}$ The energy of the Compton scattered photon is approximated by:

$$
k=k_{\text {free }}\left(1-\frac{p_{z}\left|\boldsymbol{k}-\boldsymbol{k}_{\mathbf{0}}\right|}{m_{0} c k_{0}}\right),
$$

where $k_{\text {free }}$ is the standard Compton formula:

$$
k_{\text {free }}=\frac{k_{0}}{1+\frac{k_{0}}{m_{0} c^{2}}(1-\cos \phi)},
$$

$k_{0}$ is the incident photon energy, $k$ is the scattered photon energy and $\phi$ is the photon scatter angle. $p_{z}$ is the component of electron momentum in the direction of the incident photon.

The probability of a Compton or Rayleigh scattering event is randomly sampled based on the respective total cross section for a particular element and incident photon energy. The cross sections are obtained by interpolation of the photon data tables in the EPDL97 library. ${ }^{11}$

The angle of the scattered photon is randomly sampled, in the case of Compton scatter, from the KleinNishina differential cross section multiplied by the incoherent scattering form factor from the EPDL97 library. In the case of Rayleigh scatter, the scatter angle is sampled from the Rayleigh formula $\left(1+\cos ^{2} \phi\right) \sin \phi$ multiplied by the coherent scattering form factor from the EPDL97 library.

\section{SIMULATED OBJECTS}

Four objects, displayed in Fig. 3, were simulated to analyse the effects of an uncollimated detector x-ray CT machine in airport baggage scanning and medical imaging. The Body Phantom is a $15 \mathrm{~cm}$ radius cylinder of water. The Brain Phantom consists of a $9 \mathrm{~cm}$ radius cylinder, where the outer centimetre is made of bone and the inner $8 \mathrm{~cm}$ is made of water. The Cotton Suitcase is a box with a $2 \mathrm{~mm}$ rim of PVC and an inner box of dimensions $45 \mathrm{~cm} \times 25 \mathrm{~cm}$ containing cotton, with a density of $0.2 \mathrm{~g} / \mathrm{cm}^{3}$. The Mixed Suitcase is the same as the Cotton Suitcase but with two additional objects. A $0.5 \mathrm{~mm}$ thick ring of aluminium with an inner radius of $2.95 \mathrm{~cm}$ consisting of butane gas and a $1 \mathrm{~mm}$ thick square container of PVC containing a $2.9 \mathrm{~cm} \times 2.9 \mathrm{~cm}$ square of water. 


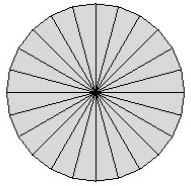

(a) Body Phantom: $15 \mathrm{~cm}$ radius cylinder of water

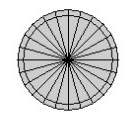

(b) Brain Phantom: $8 \mathrm{~cm}$ (c) Cotton Suitcase: $45 \mathrm{~cm}$ radius water cylinder sur- $\mathrm{x} 25 \mathrm{~cm}$ box of cotton with a rounded by $1 \mathrm{~cm}$ of bone
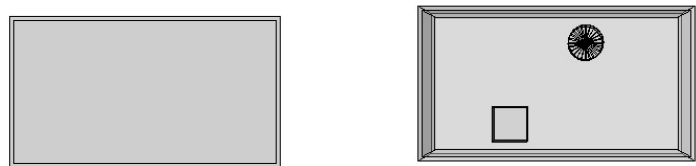

Figure 3: VRMLview images of Scattering objects.

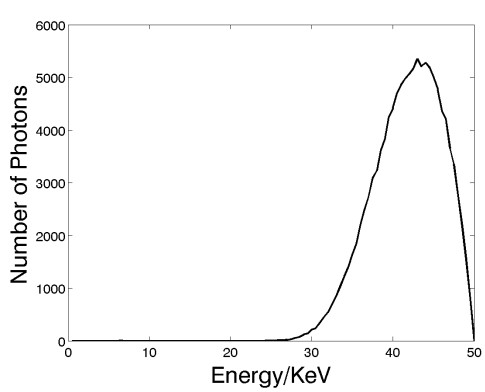

(a) $50 \mathrm{kVp}$

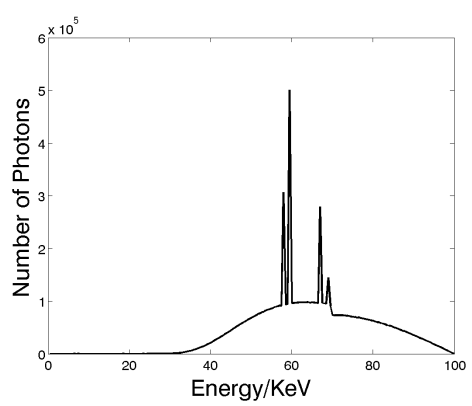

(b) $100 \mathrm{kVp}$

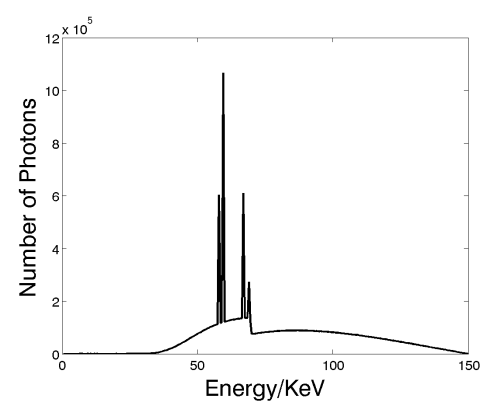

(c) $150 \mathrm{kVp}$

Figure 4: X-ray Input Spectra.

\section{EXPERIMENTAL SETUP}

The results were collected based on $50 \mathrm{kVp}, 100 \mathrm{kVp}$ and $150 \mathrm{kVp}$ input $\mathrm{x}$-ray spectra for a tungsten target. ${ }^{12}$ The input spectrum is modified by the attenuation due to the x-ray source vacuum housing and the resulting spectrum used in the simulation at each of these energies is displayed in Fig. 4.

One source projection and one ring of detectors were considered. The detectors lie on straight-line segments with the centre of each segment lying on a circle of radius $46 \mathrm{~cm} .17000$ photons per detector were transmitted from a randomly sampled point on the source to a randomly sampled point in the active detector region. The number of photons was based on real data collection. Particle cut rates were set to $1.0 \AA$.

All four objects were analysed for each x-ray spectrum. The objects were placed at the centre of the scanning region and material properties were taken from the internal Geant4 materials database, which is derived from the National Institute of Science and Technology (NIST) database. ${ }^{13}$

Each simulation took between 3 and 8 hours to complete on one processor with a clock rate of $2 \mathrm{GHz}$, depending on the size and material of the object.

\section{RESULTS}

Fig. 5 displays the results obtained for the Body Phantom with the 100kVp input spectrum. At lower energies and small angles Rayleigh scatter is expected to be dominant, where as at higher energies and wider angles Compton scatter is expected to dominate. This is clearly the case in this instance, which illustrates that the scattering processes behave as expected. 


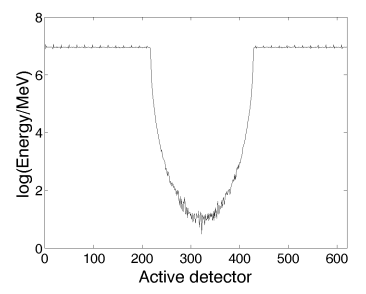

(a) Primary Photons

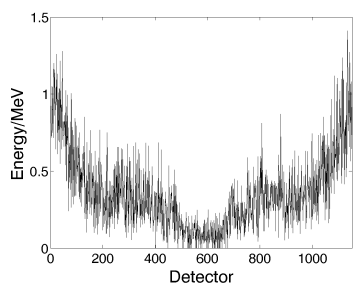

(b) Total Scatter

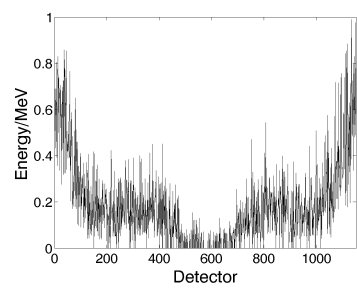

(c) Compton Scatter

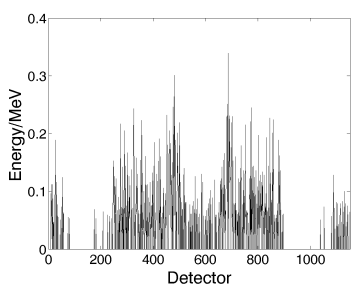

(d) Rayleigh Scatter

Figure 5: Simulation results for the Body Phantom at $100 \mathrm{kVp}$.

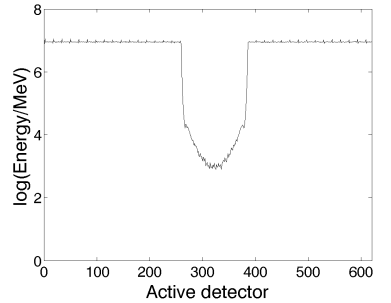

(a) Primary Photons

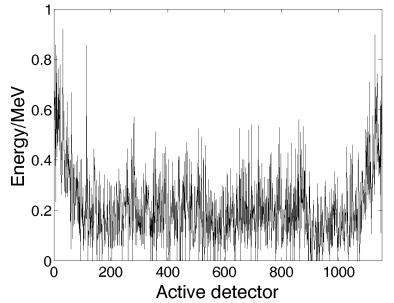

(b) Total Scatter

Figure 6: Simulation results for the Brain Phantom at $100 \mathrm{kVp}$.

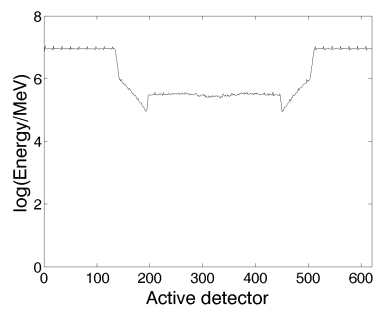

(a) Primary Photons

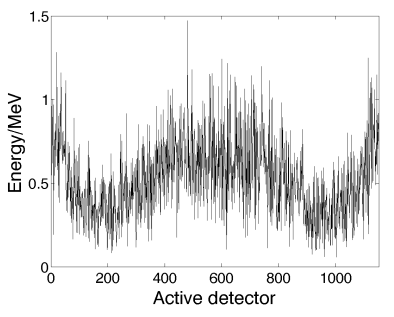

(b) Total Scatter

Figure 7: Simulation results for the Cotton Suitcase at $100 \mathrm{kVp}$.

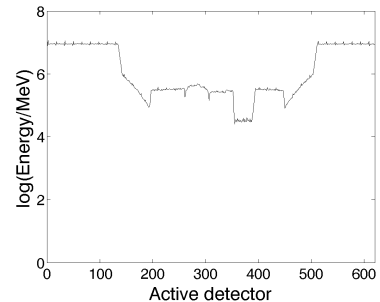

(a) Primary Photons

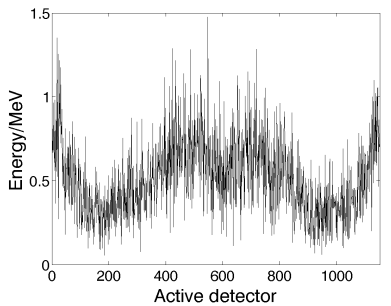

(b) Total Scatter

Figure 8: Simulation results for the Mixed Suitcase at $100 \mathrm{kVp}$. 
Table 1: Comparision of Scatter Contributions

\begin{tabular}{c|ccc}
\hline & \multicolumn{3}{|c}{ Max Scatter Contribution (\%) } \\
\hline Object & $50 \mathrm{kVp}$ & $100 \mathrm{kVp}$ & $150 \mathrm{kVp}$ \\
\hline Body Phantom & 37.21 & 8.72 & 6.77 \\
Brain Phantom & 4.72 & 1.51 & 1.24 \\
Cotton Suitcase & 2.18 & 0.8 & 0.56 \\
Mixed Suitcase & 6.61 & 0.99 & 0.83 \\
\hline
\end{tabular}

Table 2: Single and multiple scatter contribution as a percentage of total scatter

\begin{tabular}{c|ccc||ccc}
\hline & \multicolumn{3}{|c||}{ Single (\%) } & \multicolumn{3}{c}{ Multiple (\%) } \\
\hline Object & $50 \mathrm{kVp}$ & $100 \mathrm{kVp}$ & $150 \mathrm{kVp}$ & $50 \mathrm{kVp}$ & $100 \mathrm{kVp}$ & $150 \mathrm{kVp}$ \\
\hline Body Phantom & 69.57 & 60.24 & 55.14 & 30.43 & 39.76 & 44.86 \\
Brain Phantom & 90.63 & 78.50 & 76.90 & 9.37 & 21.49 & 23.10 \\
Cotton Suitcase & 75.35 & 77.19 & 77.16 & 24.64 & 22.81 & 22.84 \\
Mixed Suitcase & 58.96 & 75.85 & 75.87 & 41.04 & 24.15 & 24.13 \\
\hline
\end{tabular}

Fig. 6 8 illustrate the results obtained for the Brain Phantom, Cotton Suitcase and Mixed Suitcase respectively for $100 \mathrm{kVp}$. The Body and Brain Phantoms are more attenuating than the suitcases since water is more dense than cotton. The Body Phantom is more attenuating than the Brain Phantom since it has a larger radius creating a longer path for the photons to travel through the object.

The total energy of scattered photons recorded in each detector for the two suitcase objects is generally higher than the scattered energy recorded for the Body and Brain Phantoms.

The fractional contribution of scatter to the image is found by dividing the total scatter recorded in each detector by the total energy recorded. The scatter contribution for each object and each input spectrum are shown in Fig. 9 and Table 1 displays the maximum percentage of scatter contributing to the image for this projection.

Scatter levels are found to be highest for each of the objects when the lowest input spectrum $(50 \mathrm{kVp})$ is used. Low energy photons are highly absorbing so less primary beam is recorded. The Body Phantom projection contains very high levels of scatter, reaching $37 \%$ in this instance.

For each input spectrum the Body Phantom projection contains the highest percentage of scatter. As the x-ray energy of the input spectrum increases the levels of scatter contributing to the image decrease for each object considered. For $100 \mathrm{kVp}$ and $150 \mathrm{kVp}$ both of the suitcase objects have scatter levels of less than $1 \%$.

A number of algorithms have been proposed for the removal of scatter from reconstructed x-ray CT images. These algorithms are usually based on single scatter approximations as it is possible to determine the location of the scatterer in this instance. When x-rays have been scattered multiple times, the origins of the scattering event are unknown. Table 2 shows a breakdown of the total scatter into single and multiple contributions.

\section{DISCUSSION AND CONCLUSION}

For medical applications, an uncollimated x-ray CT machine could lead to high levels of scatter in the image if the object being scanned was large. For a large water based object, such as the human body, most of the primary beam in the shadow of the object is absorbed, leaving a high level of the recorded data due to scattered photons. Advanced scatter correction methods would be required to reconstruct an acceptable image.

Since the majority of space in a suitcase typically consists of clothing, which has a very low density, scatter contribution compared to primary beam is negligible. In baggage scanning, identifying certain materials is a priority and so it is important to ensure accuracy in reconstructed density. These results indicate that since 

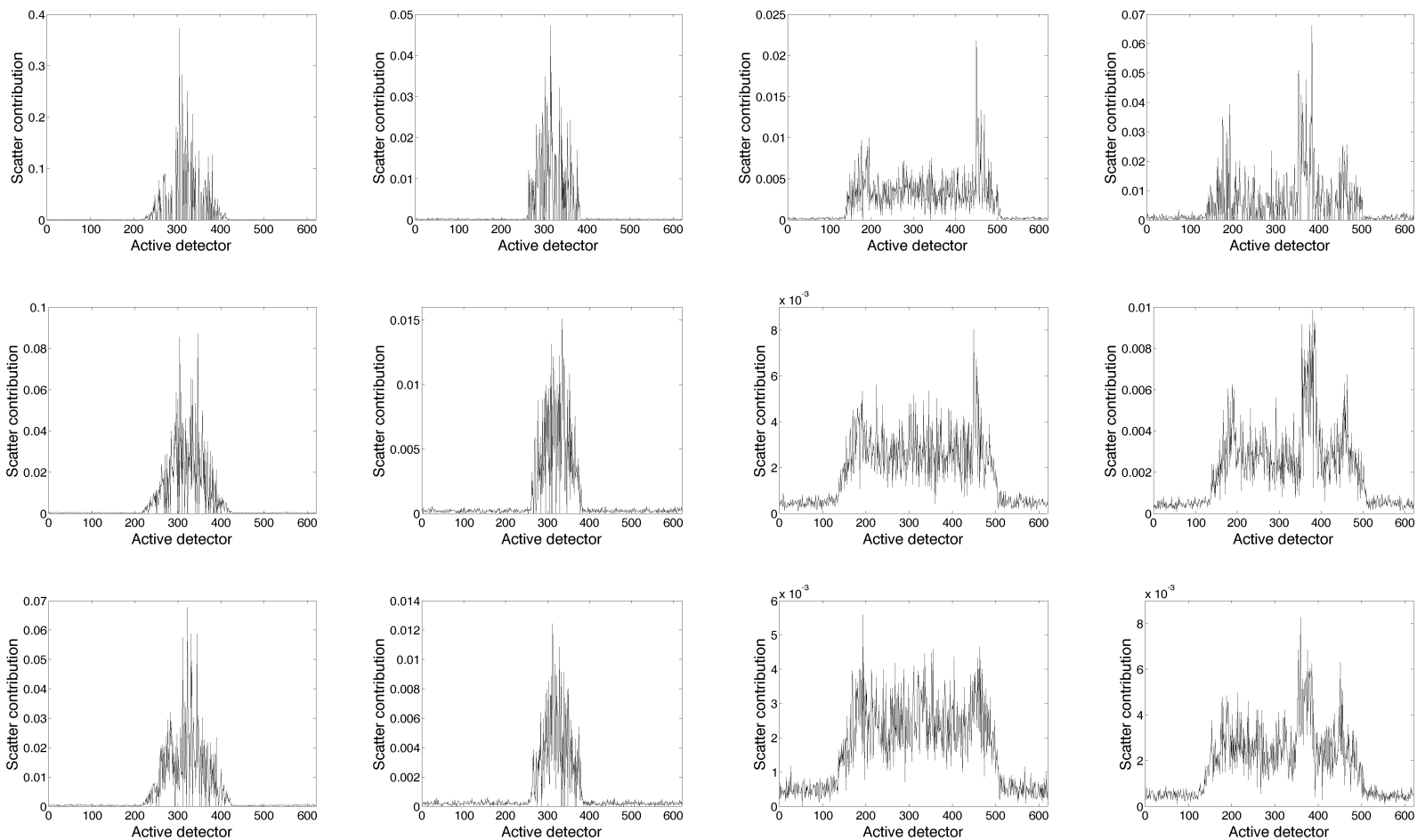

(a) Body Phantom

(b) Brain Phantom

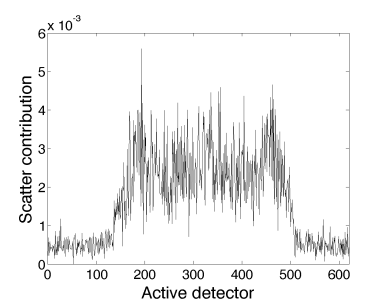

(c) Cotton Suitcase

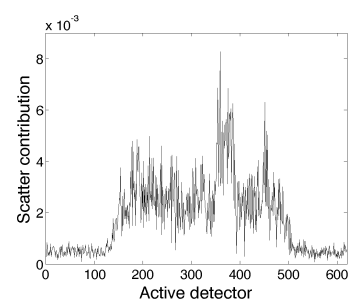

(d) Mixed Suitcase

Figure 9: Scatter contribution to the image. The first row illustrates the scatter contribution for each object with a $50 \mathrm{kVp}$ input spectrum, the second row with $100 \mathrm{kVp}$ input spectrum and the third row with $150 \mathrm{k} \mathrm{V}$ input spectrum.

the contribution of scattered energy is low in baggage scanning applications compared to medical scenarios, the absense of detector collimation does not unduly compromise image quality in this instance.

A comparison with experimental results is underway.

\section{ACKNOWLEDGMENTS}

We are grateful to EPSRC (EP/E010997/1) and to Rapiscan Systems Ltd for their financial support of this work.

\section{REFERENCES}

[1] Rapiscan Systems, "Rapiscan Real Time Tomography", http://www.rapiscansystems.com/real-time-tomography/real-time-tomography.html, 2009.

[2] E. Cook, R. Fong, J. Horrocks, D. Wilkinson, R. Speller, "Energy dispersive X-ray diffraction as a means to identify illicit materials: A preliminary optimisation study", Applied Radiation and Isotopes Vol. 65, no. 8, pp. 959-967, 2007.

[3] P.C. Johns, M. Yaffe, "Scattered radiation in fan beam imaging systems", Med. Phys. 9(2), 1982.

[4] G.H. Glover, "Compton scatter effects in CT reconstructions", Med. Phys. 9(6), 1982.

[5] P. M. Joseph, R. D. Spital, "The effects of scatter in x-ray computed tomography", Med. Phys. $28(2), 2001$.

[6] C. H. Malden, R. D. Speller, "A CdZnTe array for the detection of explosives in baggage by energy-dispersive X-ray diffraction signatures at multiple scatter angles", Nucl. Instr. Meth. Phys. Res. A, Vol. 449, pp. 408-415, 2000. 
[7] S. Agnostinelli et al., "GEANT4 - a simulation toolkit", Nucl. Instr. Meth. Phys. Res. A, Vol 506, no. 3, pp. 250-303, 2003.

[8] R. Marc Kippen, "The GEANT4 low energy Compton scattering (G4LECS) package for use in simulating advanced Compton telescopes", New Astron. Rev. 48, pp. 221-225, 2004.

[9] J. H. Siewerdsen, D. A. Jaffray, "Cone-beam computed tomography with a flat-panel imager: Magnitude and effects of x-ray scatter", Med. Phys. 28(2), 2001.

[10] R. Ribberfors, "Relationship of the relativistic Compton cross section to the momentum distribution of bound electron states", Phys. Rev. B, Vol. 12, no. 6, 1975.

[11] D. E. Cullen, J. H. Hubbell, L. Kissel, "EPDL97: the Evaluated Photon Data Library, '97 Version", UCRL50400, Vol. 6, Rev. 5, 1997.

[12] K. Cranley, B. J. Gilmore, G. W. A. Fogarty, L. Desponds, D. Sutton, "Catalogue of Diagnostic X-Ray Spectra \& Other Data", Institute of Physics and Engineering in Medicine, Report 78.

[13] J. S. Coursey, D. J. Schwab, R. A. Dragoset, Atomic Weights and Isotopic Compositions (version 2.4.1), 2005. [Online] Available: http://physics.nist.gov/Comp [2009, April 11]. National Institute of Standards and Technology, Gaithersburg, MD. 